\title{
THE PHYSICS OF CARBON DUST AROUND WC STARS
}

\author{
V.G. ZUBKO \\ Main Astronomical Observatory, Kyjiv, NAS Ukraine
}

\begin{abstract}
The physical properties of dust in WR shells are investigated in detail. It has been found that carbon grains may grow in the severe conditions of WR shells due to collisions with impinging positive carbon ions. Detailed physical models of carbon dust and dust production rates for selected WC stars have been calculated. It is concluded that graphite rather than amorphous carbon is the most suitable grain material.
\end{abstract}

Key words: stars: Wolf-Rayet - circumstellar dust - infrared radiation

\section{Introduction}

The problem of carbon dust formation and survival is still one of the most intriguing problems in the WR star astrophysics (see e.g., van der Hucht 1992). For WC stars with permanent dust production Williams et al. (1987) have proposed simple models of dust shells under the assumptions of spherical symmetry, an inverse square law for the dust density distribution and amorphous carbon as the dust constituent. A suitable mechanism for the carbon grain growth under the conditions of practically full carbon ionization has been offered and modelled by Zubko et al. (1992): a grain moving relative to the wind may grow due to collisions with incident carbon ions. Moreover, Zubko (1992) has developed a theory of the micro-structure of a steady-state WR star dust shell on the base of the kinetic equation for the dust grain distribution function. For the special case of a geometrically thin nucleation layer this theory has been applied to model and fit the spectra of some individual WC stars. Initially, we had to make some simplifications for the equations for the dust grain parameters. The results described below make use of more exact equations. Here we recalculated the best-fit models of the dust shells of some WC9-10 single stars.

\section{Formulation of the dust shell model}

We assumed stationarity and spherical symmetry; also a constant velocity of the plasma shell outflow $v$ and, as a consequence, an inverse square law for the number density distributions of all plasma components. Three chemical elements have been taken into account: $\mathrm{He}$ (in the form of the $\mathrm{He}^{+}$ion), $\mathrm{C}$ $\left(\mathrm{C}^{++}\right)$and $\mathrm{O}\left(\mathrm{O}^{++}\right)$. We assumed constancy of the ionization state distributions throughout the model, in accord with the results of van der Hucht $e t$ al. (1986). The dust shell is optically transparent in both ultraviolet (where the star emits) and infrared regions. We assume the existence of a single nucleation layer where the condensation nuclei are formed (the nature of the nucleation process is still unknown). The depth of this layer is small in comparison with the distance from the star $R$. The parameter $R$ is the one varied 
to reach the best fitting. Then the number density of grains $n$ at the distance $R$ may be evaluated from the continuity equation: $n=J / 4 \pi R^{2}(v+u)$ where $J$ is the condensation nuclei production per time unit and $u$ is the drift velocity of a dust grain. The thermal balance of dust grains in the WC shell is determined mainly by the absorption of stellar ultraviolet radiation and infrared re-emission. We have used for the dust temperature calculations the thermal balance equation (5) from Zubko (1992) (see, also, Spitzer 1978). The role of collisions is negligible. The electric charge equilibrium for the dust grain is determined by the balance of the photoelectric effect caused by the stellar ultraviolet radiation and collisions with electrons and carbon ions. We have used for the dust electric potential calculations the equation (4) from Zubko (1992) (see, also, Draine \& Salpeter 1979). The dust grain dynamics is dominated by a stellar radiation pressure and a drag force. We used an equation for the steady grain drift velocity $u$ because it relaxes very quickly. To calculate a drag force we have used formulae (5-6) from the work of Dwek \& Arendt (1992). A drag force contains two terms. The first one takes into account the direct collisions with the gas particles and the second is defined by the cumulative effect of small-angle Coulomb scattering on a charged dust grain moving through an ionized gas.

For the dust grain growth calculations we have taken into account grain growth due to collisions with incident carbon ions including the effects of the drift velocity and the dust electric charge and dust destruction due to the thermal evaporation and the kinetic sputtering by all the possible species. The main formulae have been taken from the work of Draine \& Salpeter (1979). We have calculated the dust shell luminosity $L_{\lambda}$ from the relation $L_{\lambda}=J L_{\lambda}^{\prime}(\infty)$, where $L_{\lambda}^{\prime}(R)$ obeys the equation

$$
\frac{d L_{\lambda}^{\prime}}{d R}=\frac{4 \pi a^{2}}{v+u} Q_{\lambda}\left(a, T_{d}\right) \pi B_{\lambda}\left(T_{d}\right)
$$

To control the type of carbon growing on a dust grain (graphite or amorphous carbon) we have used a physical criterion of Gail \& Sedlmayr (1984) modified by Zubko (1992) in a form suitable for the present problem.

\section{Results of modelling}

We have modelled the IR-spectra of six single late WC stars: WR69, WR76, WR80, WR95, WR96 and WR118 making use of the corresponding data of Williams et al. (1987). We have adopted the following values of the main parameters: $v=2000 \mathrm{~km} / \mathrm{s}, T=6000 \mathrm{~K}, M_{*}=8 \times 10^{-5} \mathrm{M}_{\odot} / \mathrm{yr}, R_{*}=$ $10 \mathrm{R}_{\odot}$, chemical composition (by mass): $\mathrm{C} / \mathrm{He}=0.9,0 / \mathrm{He}=0.08, T_{*}=$ $22000 \mathrm{~K}$ for WC9 stars and $20000 \mathrm{~K}$ for WC10 stars, and the radius of condensation nuclei equals $0.4 \mathrm{~nm}$. The varied parameter was the distance to the nucleation layer $R_{0}$. The fitting technique has been described by Zubko (1992). The main results of our calculations are presented in Table I. 
TABLE I

The calculated parameters of some WC star dust shells

\begin{tabular}{rrcccccc}
\hline WR & $R_{o}$ & $T_{o}$ & $a_{f}$ & $J$ & $\dot{M}$ & $d / g$ & $m$ \\
\hline 69 & 850 & 1551 & 8.0 & $3.1+34$ & $2.4-9$ & 0.01 & 5.7 \\
76 & 1365 & 1308 & 8.0 & $7.7+36$ & $5.9-7$ & 1.71 & 4.1 \\
80 & 1080 & 1422 & 8.0 & $2.5+35$ & $1.9-8$ & 0.06 & 5.0 \\
95 & 850 & 1551 & 8.0 & $7.7+34$ & $5.9-9$ & 0.02 & 4.0 \\
96 & 880 & 1531 & 8.0 & $1.9+35$ & $1.4-8$ & 0.04 & 4.0 \\
118 & 975 & 1346 & 13.4 & $1.8+36$ & $6.4-7$ & 1.85 & 4.0 \\
\hline
\end{tabular}

Notes. WR numbers from the Catalogue of WR stars by van der Hucht et al. (1981); $R_{o}$ : nucleation distance in $R_{*} ; T_{o}$ : dust temperature at $R_{o}$ in $\mathrm{K} ; a_{f}$ : final size of a dust grain in $\mathrm{nm} ; J$ : nuclei condensation rate in $1 / \mathrm{s} ; \dot{M}$ : dust mass loss in $\mathrm{M}_{\odot} / \mathrm{yr} ; d / g$ : carbon dust/gas ratio in $\% ; m$ : fitting error in $\%$.

TABLE II

The dust shell model for a typical WC9 star (WR95)

\begin{tabular}{rcrcrrr}
\hline$R$ & $a$ & $T_{d}$ & $u$ & $\Phi$ & $1-Y$ & $\log \left(t_{c} / t_{h}\right)$ \\
\hline 850 & 0.40 & 1551 & 15.1 & 1.1 & 1.00 & 6.1 \\
896 & 1.38 & 1521 & 25.3 & 5.6 & 1.00 & 4.7 \\
947 & 2.86 & 1493 & 40.7 & 10.2 & 0.91 & 3.8 \\
1038 & 5.54 & 1446 & 61.1 & 13.3 & 0.45 & 3.1 \\
1140 & 6.97 & 1396 & 69.3 & 14.0 & 0.18 & 3.0 \\
2103 & 7.95 & 1118 & 74.0 & 14.2 & 0.00 & 2.6 \\
3990 & 7.98 & 889 & 74.1 & 14.2 & 0.00 & 0.0 \\
11138 & 8.00 & 615 & 74.0 & 14.2 & 0.00 & -6.7 \\
20766 & 8.01 & 494 & 74.0 & 14.2 & 0.00 & -12.2 \\
104505 & 8.02 & 276 & 73.8 & 14.2 & 0.01 & -34.4 \\
\hline
\end{tabular}

Notes. $R$ : distance to the star in $R_{*} ; a$ : dust grain radius in $\mathrm{nm} ; T_{d}$ : dust grain temperature in $\mathrm{K} ; \boldsymbol{u}$ : drift velocity of a dust grain in $\mathrm{km} / \mathrm{s} ; \Phi$ : dimensionless electric energy of a dust grain; $1-Y$ : the measure of kinetic sputtering; $\log \left(t_{c} / t_{h}\right)$ : the ratio of the characteristic time-scales of condensation $t_{c} / t_{h}$, where $t_{h}$ is the surface hopping time and $t_{c}$ is the mean capture time (Gail \& Sedlmayr 1984; Zubko 1992).

Dust shell models for a typical WC9 star (WR95) and WC10 star (WR118) are presented in Tables II and III, respectively. For all the best-fit models $t_{c} / t_{h}>1$ in the zone of intensive dust grain growth and where the dust IRradiation is mainly formed. As a consequence graphite dust grains should start to grow. However, at greater distances from the star an amorphous carbon mantle may grow as well. As a rule the mantle is too thin to produce a visible effect in the dust shell. 
TABLE III

The dust shell model for the WC10 star (WR118)

\begin{tabular}{rrrcrrr}
\hline$R$ & $a$ & $T_{d}$ & $u$ & $\Phi$ & $1-Y$ & $\log \left(t_{c} / t_{h}\right)$ \\
\hline 975 & 0.40 & 1346 & 12.4 & 0.0 & 1.00 & 4.9 \\
1009 & 0.88 & 1328 & 18.0 & 1.2 & 1.00 & 4.0 \\
1066 & 1.87 & 1304 & 25.8 & 3.4 & 1.00 & 3.0 \\
1157 & 3.74 & 1267 & 37.1 & 7.0 & 0.94 & 2.0 \\
1211 & 4.90 & 1248 & 43.3 & 8.7 & 0.87 & 1.6 \\
1500 & 9.40 & 1153 & 62.2 & 11.1 & 0.41 & 0.4 \\
1710 & 10.86 & 1098 & 66.8 & 11.2 & 0.25 & -0.1 \\
2226 & 12.25 & 995 & 70.7 & 11.2 & 0.11 & -1.0 \\
10720 & 13.32 & 565 & 73.3 & 11.2 & 0.02 & -10.0 \\
106667 & 13.40 & 253 & 73.3 & 11.2 & 0.02 & -42.4 \\
\hline
\end{tabular}

\section{Acknowledgements}

I am grateful to the International Astronomical Union and the International Science Foundation (grant N1261/4) for financial support.

\section{References}

Draine, B.T., Salpeter, E.E. 1979, ApJ 231, 77

Dwek, E., Arendt, R.G. 1992, Ann. Rev. $A \mathcal{G} A$ 30, 11

Gail, H.-P., Sedlmayr, E. 1984, $A \& A$ 132, 163

van der Hucht, K.A., Conti, P.S., Lundström, I., Stenholm, B. 1981, Sp. Sci. Rev. 28, 227

van der Hucht, K.A., Cassinelli, J.P., Williams, P.M. 1986, $A \& A$ 168, 111

van der Hucht, K.A., 1992, The AEA Rev. 4, 123

Spitzer, L. 1978, Physical Processes in the Interstellar Medium (New York: Wiley)

Williams, P.M., van der Hucht, K.A., Thé, P.S. 1987, $A \& A$ 182, 91

Zubko, V.G., Marchenko, S.V., Nugis, T. 1992, Astron. Astrophys. Trans. 3, 131.

Zubko, V.G. 1992, Astron. Astrophys. Trans. 3, 141

\section{DISCUSSION:}

Cherchneff: Don't you think that the size of your condensation nuclei is a bit small? A $4 \AA$ radius rather characterizes a molecule than a grain. Therefore, your growing process is not truly adequate for such a small condensation nucleus.

Zubko: I have to choose some artificial (more or less plausible) value for the condensation nuclei size in the intermediate region of sizes (molecule-bulk body) because of the lack of a suitable mechanism for grain nucleation at the WC winds. Such a choice may be partly justified by one of the results of the present work that the final sizes of dust grains in the calculated models are considerably larger in comparison with the adopted nuclei radius (8.0-13.0nm). 